\title{
Comparative Analysis of dyeing wastewater treatment Technology
}

\author{
Guihong $P E I^{1,}$, Feng $\mathrm{YU}^{1, \mathrm{~b}}$ and Liyin $Z \mathrm{HANG}^{1, \mathrm{c}}$ \\ ${ }^{1}$ School of Civil Engineering and Architecture, Southwest Petroleum University, Chengdu China \\ a570769297@qq.com, ${ }^{b} 1010468261 @ q q . c o m,{ }^{c} 377231918 @ q q . c o m$
}

\begin{abstract}
Keywords: sewage treatment, environmental engineering, wastewater, oxidation
Abstract. Dyeing wastewater complex composition, color large organic pollutant concentration is very high, in the field of wastewater treatment is a kind of low biodegradability, biodegradation of industrial wastewater. Based on extensive research, printing and dyeing wastewater treatment method commonly used in the classification, and analyzes the advantages and disadvantages of each treatment and the effect of each treatment method, research has certain reference value for dyeing wastewater treatment process selection.
\end{abstract}

\section{Introduction}

Dyeing wastewater mainly refers to cotton, linen, chemical fiber and other fabrics produced in the process wastewater, including integrated production process waste water emissions from each section. Different processing methods and the role of each section, the effluent water quality is different, specific processing procedures desizing, scouring, bleaching, mercerizing, dyeing, printing and finishing section. Desizing wastewater major pollutants from the use of polyvinyl alcohol and starch sizing, so high COD, whose value is up to $10000 \mathrm{mg} / \mathrm{L}$ or more, and biochemical oxygen demand BOD is very low, low biodegradability. Scouring waste water contains a lot of scouring process residual alkali, alkaline water quality big, a certain amount of COD and SS, a large amount of water and high water temperature. The main purpose is to use bleach to remove fibers and strong oxidants colored impurities on the fabric, increase the whiteness of the fiber surface, high impurity content of waste water. As the name suggests is a silk fiber to handle the increased surface gloss, the surface more friendly process under certain conditions of tension on the fiber surface treated with lye, silk waste alkaline. Bleached and mercerized by the process, the fiber surface becomes smooth affinity, easy to carry out dyeing. Dyeing process is more complex, the purpose is to make the surface of the fabric color, and the dye attached to the fabric in a chemical or physical and chemical effects, or directly on the fiber surface to form the desired color by direct synthesis. According to the type of fabric and the desired color is different, very different dyeing materials and methods used in making qualitative changes in wastewater pollutants in water and the water is very high. Printing process by the printing cylinder, screen printing and other equipment to the textile surface of the slurry, and subsequent fixation, soaping process, waste water mainly from equipment washing waste water and soap. Printing station, in addition to waste water contains a lot of pigment and additives, as well as a large number of printing paste, COD and BOD of wastewater are high. Waste water discharge finishing section of a small impurity content, but lint, resins, oils and other very high.

Under normal circumstances dyeing wastewater color and COD from most fiber, dyes, additives and textile raw materials contained in the slurry. Printing process for each section in different ways, their dyes, additives, and other pulp not completely attached to the fabric, the residual chemicals in the water increases the concentration of pollutants in wastewater and chroma machine. The quality of wastewater generated by different processes are different, such as scouring desizing wastewater and the wastewater contains large amounts of COD, mercerizing process requires a large amount of alkali, alkaline content of large waste. Different types of fabrics and materials and processes, the different pollutants, wastewater quality vary greatly. Dyeing and printing processes, in order to achieve a better effect of the stain requires at different $\mathrm{pH}$ conditions printing, therefore, the $\mathrm{pH}$ value of the difference between the discharge station corresponding to a large water. Normally, cotton and blended fabrics need to avoid high alkalinity was printed, so the wastewater is another feature large water alkalinity and 
high $\mathrm{pH}$ values. Quantity changes is also a feature of printing and dyeing wastewater, due to the different business processes and printing processes, printing and dyeing enterprises only partially discharged wastewater, but there are still some companies wastewater and domestic sewage discharged together, so the quantity and quality of printing and dyeing wastewater constantly changing. Printing and dyeing wastewater and water quality characteristics of many factors, such as fabric varieties and yield, the type and amount of dye.

Dyeing wastewater in wastewater treatment is a kind of low biodegradability, biodegradation of industrial wastewater. Textile industry based on the use of different raw materials products, printing and dyeing wastewater generated are not the same, usually dyeing wastewater $\mathrm{pH}$ of 6-10, COD is 400-2500mg / L, BOD 5 of 100-400mg / L, SS 100 to 200mg / L, chromaticity of 100-500 times.

\section{Common Dyeing Wastewater Treatment Methods}

Dyeing wastewater treatment methods commonly used method can be divided into physical, chemical oxidation, biochemical, and other advanced oxidation processes combined treatment method. Physical laws generally apply to precipitation, coagulation, floating, adsorption, flotation, filtration and the like. Chemical treatment including neutralization, electrolysis, redox, ion exchange, electrodialysis. Biochemical rule is the use of microbial metabolism, the wastewater organic pollutants into stable and harmless substances, which have the most common anaerobic oxidation, aerobic aerobic, anaerobic aerobic.

(1) Physical treatment

Dyeing wastewater chemical method refers to the removal of contaminants by physical ways, the main common ways include precipitation, grille, filtration, adsorption, and reverse osmosis. Chemical method has simple process, easy operation, low cost, obvious effect.

Hukun Wang et[1] alone dyeing wastewater treatment with activated carbon, COD and chroma adsorption effect is obvious, respectively, $74.9 \%$ and $77.1 \%$. Wang Daizhi[2] generation media Pupil use of fly ash adsorption of dyeing wastewater treatment, results showed that the effluent chroma reduced significantly. Adsorption Treatment of dyeing wastewater, decolorization found good results. Adsorption HDTMA- bentonite, waste water treatment and applied to good effect. AVIATION cotton soil as raw material after calcination to obtain new efficient dyeing wastewater decolorizing materials, more than $90 \%$ of wastewater decolorization rate.

(2) Membrane separation

Membrane separation technology was first developed in the 1960s began to be used fresh water purification project in recent years, film printing and dyeing wastewater treatment has been widely used, membrane separation technology is based on the existence of a pressure difference on both sides of the membrane, the vapor partial pressure, concentration poor, poor thrust under the effect of the potential of the selected permeability characteristics of the membrane of the impurities in the waste water separation, purification and enrichment methods. Membrane separation process can be divided into microfiltration, ultrafiltration, nanofiltration and reverse osmosis and other forms of microfiltration membrane separation generally having a diameter of 0.05 to 20 microns large impurities, usually used in ultrafiltration, nanofiltration pretreatment. Viruses such as water, the colloid so can be removed. Nanofiltration major diameter of about $1 \mathrm{~nm}$ to remove impurities, mostly because of its charged composite membrane, so its easy separation by electrical effects of the solution. Nanofiltration in water COD, removal efficiency of chroma well. Reverse osmosis membrane pore size of about 0.1 to $1.0 \mathrm{~nm}$, their effect on ion intercept the water well at a depth of wastewater treatment in the use of reverse osmosis. Membrane separation technology has no phase change, simple equipment, easy operation, with good prospects for development, it is currently used for advanced treatment of wastewater, the effect when used in combination with other techniques excellent.

Qin[6] applied nano membrane to dyeing wastewater treatment, water works well, most of the water back up to the criteria used. Inorder to inspect the microfiltration - Nanofiltration pretreatment means secondary clarifier effluent color removal, results showed: effluent COD removal efficiency 
greater than $86 \%$ rejection rate is higher than $95 \%$. A new textile polyvinyl Microfiltration wastewater treatment efficiency was good, COD removal efficiency of $75 \%$. Ultrafiltration-reverse osmosis membrane separation and wastewater treatment applications in the textile industry, engineering practice shows that the COD removal efficiency of $99 \%$, stable operation, wastewater reuse rate of $85 \%$. Barredo-Damas $\mathrm{TiO}_{2}-\mathrm{ZrO}_{2}$ such as the use of ultrafiltration textile organic matter in wastewater, COD removal efficiency up to $79 \%$ color removal rate can be $98 \%$ greater than $99 \%$ turbidity removal.Micro omentum bioreactor treatment of dyeing wastewater, the reactor effluent water quality is good, COD removal rate of $85 \%$.

(3) Chemical Treatment Method

Common chemical dyeing wastewater treatment methods include coagulation, electrolysis and micro-electrolysis method, photocatalytic oxidation, Fenton oxidation, ozone oxidation method.

Zhang Yan[12] treatment dyeing wastewater by coagulation and biological applications in practical engineering, the effluent wastewater can be an emission standard. Coagulation process of dyeing waste degradation were studied using $\mathrm{Al}_{2}\left(\mathrm{SO}_{4}\right)_{3} \cdot 18 \mathrm{H}_{2} \mathrm{O}$ as a flocculant, the optimum amount of coagulant is $2.5 \mathrm{~mL} / \mathrm{L}$, dyeing wastewater effluent best.The new flocculant KD-800 and polyacrylamide combination process, the degradation of the test reactive dyeing wastewater, decolorization and studied factors. Gao Meng[15] use high coagulant ferric sulfate (PFS) of dyeing wastewater was investigated PFS used alone and in combination with polypropylene ether amine (PAM) effect was found when PFS and PAM combined treatment is better.In the SE Study of coagulation coagulant effect practical application, and several other coagulant compared the experimental results, the results show that the dosage of $155 \mathrm{mg} / \mathrm{L}$, SE coagulation effect is best. Chen Ling[17] poly aluminum iron (II) used in dyeing wastewater treatment experiment magnesium, SS, COD and chroma removal rates were $87.9 \%, 84.2 \%$ and $95.7 \%$.

Lv Yanfei[18] using Fenton reagent degradation of acid red $\mathrm{B}$ dye wastewater, the results showed that: $\mathrm{H}_{2} \mathrm{O}_{2}$ concentration is $0.03 \mathrm{~mol} / \mathrm{L}$, the concentration of $\mathrm{H}_{2} \mathrm{O}_{2}$ and $\mathrm{Fe}^{2+}$ ratio of 20: 1, the wastewater COD removal rate was $62.32 \%$. History of red incense study the Fenton reagent treatment of dyeing wastewater, while The influence of treatment on Wastewater and COD, color removal under optimal conditions more than 95\%,. Chen Wensong et al. studied the low amount of Fenton oxidation - coagulation process of oxidation, results showed: Fenton oxidation - coagulation process complex composition of dye wastewater treatment has a good effect. Zhang Genlin[20] experiment showed homogeneous Fenton-Oxidation Process containing hydrophilic and hydrophobic dye wastewater has a good effect. Zhang Ultrasonic - Ozone combined method Decolorization of methyl orange wastewater, the results showed that the decolorization rate as ultrasonic power, ozone gas velocity increased with increasing ozone concentration, discoloring reaction of methyl orange first-order kinetics.

Adding $\mathrm{H}_{2} \mathrm{O}_{2}$ and $\mathrm{MnO}_{\mathrm{x}}-\mathrm{GAC}$ during the ozone oxidation catalyst dyeing wastewater by static test results: Hydrogen peroxide oxidation to enhance the efficiency of ozone oxidation to shorten the processing time, saving the amount of ozone. Wang Hongyang et al.[22] dyeing wastewater advanced treatment of secondary effluent when, through experiments to explore the effects of ozone depletion on the effect of water, the amount of ozone $6.5 \mathrm{mg} / \mathrm{mg}$ wastewater chroma degradation.

(4) Biological Method

The anaerobic treatment method of dyeing wastewater treatment effect, the results of continuous operation results show significantly improved biodegradability of the effluent, wastewater color removal works well, the removal rate was $77.2 \%$. Xuqi Kun and other studies of biological contact oxidation degradation of dyeing secondary effluent by BAF wastewater treatment to achieve a standard emissions. Tseng Kuo-Drive, for using ABR-SBR Combined Process wastewater, through test results show that color, $\mathrm{COD}_{5}$ removal rate was higher than $90 \%$, water works well.In regard to anaerobic-aerobic process application results in printing and dyeing wastewater, continuous operation results show that the effluent chroma and CODCr removal above $80 \%$. Pei[26] and other studies of economic feasibility Biochemical desizing wastewater treatment alone, calculated by way of example, desizing wastewater treatment method alone, more economical financially. 


\section{Conclusions}

According to the characteristics of its own textile industry, printing and dyeing wastewater treatment, should be used to repeat reuse and utilization measures, combined with textile production processes of reform, to minimize water $\backslash$ bases and other auxiliaries of the amount of wastewater dyes, pastes recovered for example, synthetic fibers and synthetic fibers containing more than $75 \%$ of the fabric using dry printing process, the production process can eliminate printing wastewater; in acidic mordant dye process, if using hydrogen peroxide or sodium nitrate potassium dichromate as the oxidant in place of the full-length, it is possible to eliminate pollution in wastewater chromium. many printing companies generally will lye for mercerizing process emissions as the process of scouring scouring liquid, scouring processes with emission of waste lye to step back paddle, repeated use can greatly reduce the total alkali content in the whole process of discharge for sewage containing sulfur can be added to the acid in the first reaction pot, so that waste water release hydrogen sulfide, and then after the precipitate is filtered recycling. Vat dyes and disperse dyes containing water, ultrafiltration may be non-water-soluble dye particles are recycled through these innovative production technology, can effectively reduce pollutant emissions from textile industry. But also for production enterprises to save a lot of raw materials, increase the economic efficiency of enterprises.

\section{References}

[1] H.K. Wang, J. Ren. Textile Auxiliaries. Vol. 25(2008), p.25

[2] D.Z. Wang. Textile Auxiliaries. Vol. 26(2009), p.32

[6] J.J. Qin, H.O.Maung, A.K. Kiran. Sepration and purification technology, Vol.56(2007), p.199

[12] Y. Zhang, and T.S. Xiao. Journal of Guangzhou University. Vol. 3(2004), p. 128

[15] M. Gao, Y.C. Chen, et al. Journal of Southwest Agriculteral University. Vol. 32(2010), p. 88

[17] L. Chen, L. Sha, et al. Technology of Water Treatment. Vol. 37(2011), p. 42

[18] Y.F. Lv. Environment Science and Technology. Vol. 22(2009), p. 63

[20] G.L. Zhang, X.J. Xu, et al. Enivironmental Protection of Chemical Industry.Vol. 26(2006), p. 38

[22] H.Y. Wang, Y.T. Guan. Enivironmental Protection of Chemical Industry. Vol. 29(2009), p. 530

[26] J.D. Di, G.Y. Pan, et al. Industrial Water Treaatment. Vol. 27(207), p. 40

[27] G.H. Pei, F. Yu and J.J. Liu. Journal of Residuals Science \& Technology. Vol. 12(2015), p.S85

[28] J.J. Liu, R. Song and M.M. Cui. Journal of Hydrodynamics. Vol. 27(2015), p. 234

[29] J.J. Liu, R. Song. Progress in Computational Fluid Dynamics, An International Journal. Vol. 15(2015), p.317

[30] D.H. Qin, L.L. Liu, J.J. Liu Materialwissenschaft Und Werkstoftechnik, Vol.46(2015), P. 978

[31] R. Song, J.J. Liu. Journal of Southwest Petroleum University, Vol.37(2015), p.138

[32] F.F. Gou, J.J. Liu. Journal of Southwest Petroleum University, Vol.37(2015), p.112

[33] Q.S. Li, H.L. Xing, J.J. Liu, and X.C. Liu. Petroleum, Vol.1(2015), p.8

[34] L. Yu, J.J. Liu. Petroleum, Vol.1(2015), p.82

[35] L.Y. Chen, J.J. Liu. Petroleum, Vol.1(2015), p.373 